\title{
EPIMORPHIC ADJUNCTION OF A WEAK ORDER UNIT TO AN ARCHIMEDEAN LATTICE-ORDERED GROUP
}

\author{
RICHARD N. BALL, ANTHONY W. HAGER, AND ANN KIZANIS
}

(Communicated by Maurice Auslander)

\begin{abstract}
It is shown that an archimedean $l$-group $G$ can be embedded into another, $H$, which has a weak unit, by an embedding that is epimorphic in archimedean $l$-groups if and only if there is countable $A \subseteq G$ with $A^{\perp}=(0)$. Then the extension $H$ can always be chosen conditionally and laterally $\sigma$ complete and the embedding essential, but can never be generated by $G$ together with finitely many extra elements unless $G$ already had a weak unit.
\end{abstract}

\section{Preliminaries}

General references for l-groups (i.e., lattice-ordered groups) are [5, 1, 13] (though this last is about vector lattices) and the recent compendium [9].

Arch denotes the category of archimedean $l$-groups with $l$-homomorphisms. We shall be exclusively concerned with Arch, though various definitions and lemmas make sense more generally. We write $G \leq H$ to mean that $G$ is a sub-l-group of $H$.

A morphism $\varepsilon: G \rightarrow H$ is an epimorphism (epic) if whenever $\alpha, \beta: H \rightarrow K$ have $\alpha \circ \varepsilon=\beta \circ \varepsilon$, then $\alpha=\beta$. An object $G$ is epicomplete if $\varepsilon: G \rightarrow H$ epic and one-to-one implies $\varepsilon$ is an isomorphism (i.e., onto). An epicompletion of $G$ is a one-to-one epic $\varepsilon: G \rightarrow H$ with $H$ epicomplete.

Needless to say, there are many nonsurjective epics in Arch. The epics in Arch are characterized in [2]; the description is a bit involved, and it shall not be used directly in this paper. A consequence of the characterization is that $G$ is epicomplete in Arch if and only if $G$ is divisible, and both conditionally and laterally $\sigma$-complete $[3,4.9]$.

For $A \subseteq G,\{g \in G|| g|\wedge| a \mid=0$ for each $a \in A\}$ is denoted $A^{\perp}$, or $A^{\perp G}$ if necessary; and $\{u\}^{\perp}$ is abbreviated to $u^{\perp}$ (or $u^{\perp G}$ ). When $u \geq 0$ and $u^{\perp}=(0)$, we call $u$ a weak unit of $G$.

$G$ is said to have the principal projection property (ppp) if for each $u \in G$, $G=u^{\perp \perp} \oplus u^{\perp}[13$, p. 136$]$; one also says, “ $G$ is projectable” $[5$, p. $142 ; 1$, p. $118]$.

A conditionally $\sigma$-complete $l$-group has ppp $[5,11.2 .3]$. Thus, any $G$ that is epicomplete in Arch has ppp.

Received by the editors June 12, 1990 and, in revised form, March 5, 1991.

1991 Mathematics Subject Classification. Primary 06F20, 18A20, 46A40.

Key words and phrases. Archimedean $l$-group, epimorphism, weak unit, coessential embedding. 
An embedding $G \leq H$ is called essential (and one also says, " $G$ is large in $H$ ") if each nonzero ideal of $H$ has nonzero intersection with $G$. This is equivalent to: if a morphism $\alpha: H \rightarrow K$ has the restriction $\left.\alpha\right|_{G}$ one-to-one, then $\alpha$ is one-to-one [7, 3.1].

In Arch, an embedding $G \leq H$ is called coessential [2, p. 12] if whenever a morphism $\alpha: H \rightarrow K$ has $\left.\alpha\right|_{G}$ identically 0 , then $\alpha$ is identically 0 . Clearly, an epic embedding is coessential (but not conversely). Coessentiality is crucial to the sequel-indeed, this paper is more about coessentiality than epicity proper-and we must elaborate.

An ideal $I$ of $H$ is an archimedean kernel if $H / I$ is archimedean. Given $S \subseteq H$, there is a least archimedean kernel $\operatorname{ak}_{H} S$ containing $S$, because Arch is closed under formation of products and sub-l-groups. Evidently, $G \leq H$ is coessential if and only if $\mathrm{ak}_{H} G=H$. We explain what that means.

In an l-group $H, h_{n} \rightarrow h$ relative uniformly (or just r.u.) if there is $v$ (called the regulator) for which: if $k \in N$, there is $n_{0}$ such that $n \geq n_{0}$ implies $k\left|h_{n}-h\right| \leq v$. For $S \subseteq H$, let $S^{\prime}=\left\{h \in H \mid\right.$ there is $\left\{h_{n}\right\}_{n \in N}$ in $S$ with $h_{n} \rightarrow h$ relatively uniformly

Then $S=\operatorname{ak}_{H} S$ if and only if $S$ is an ideal with $S^{\prime}=S$. (This appears in [13, pp. 85, 427] for vector lattices, and the proof appears to use just divisibility of $H$, but even that is easily eliminated.) It then follows that for general $S \subseteq H, \operatorname{ak}_{H} S=\bigcup_{\alpha<\omega_{1}} S_{\alpha}$, where $S_{0}$ is the ideal generated by $S$ in $H$, $S_{\alpha+1}=S_{\alpha}^{\prime}$, and for a limit ordinal $\beta, S_{\beta}=\bigcup_{\alpha<\beta} S_{\alpha}$. In summary, $G \leq H$ is coessential if and only if $H=\bigcup_{\alpha<\omega_{1}} G_{\alpha}$.

Finally, we remark that the reader's intuition for some of the constructions below might be supported by visualizing these constructions within a $D(X)$, e.g., an essential closure [7].

\section{ADJOINING A UNIT}

2.1. Theorem. In the category of archimedean l-groups, the following are equivalent about $G$ :

(a) There is $H$ with a weak unit and an epic embedding $G \leq H$.

(b) There is $H$ with a weak unit and a coessential embedding $G \leq H$.

(c) There is countable $A \subseteq G$ with $A^{\perp}=(0)$.

(d) There is epicomplete $H$ with a weak unit and an epic and essential embedding $G \leq H$.

Proof of 2.1. (a) implies (b) and (d) implies (a) are both clear.

2.2. Proposition. (a) In an archimedean l-group $H$, if $h_{n} \rightarrow h$ relatively uniformly, then $\bigcap_{n} h_{n}^{\perp} \subseteq h^{\perp}$.

(b) If $G \leq H$ is coessential in Arch, then for each $h \in H$, there is countable $A \subseteq G$ with $A^{\perp H} \subseteq h^{\perp H}$.

To prove that (b) implies (c), we apply (b) in 2.2 to a weak unit $h$, so that (0) $=h^{\perp H} \supseteq A^{\perp H} \supseteq A^{\perp G}$.

Proof of 2.2. (a) Suppose $h_{n} \rightarrow h$ r.u., say, with regulator $v$. Let $k \in \bigcap_{n} h_{n}^{\perp}$. We are to show $k \in h^{\perp}$, or, equivalently, $h \in k^{\perp}$. For $m \in N$, choose $n(m)$ for which $m\left|h_{n(m)}-h\right| \leq v$. Now $k \in h_{n(m)}^{\perp}$, equivalently, $h_{n(m)} \in k^{\perp}$, so that 
$m|h| \leq v$ modulo $k^{\perp}$, for any $m$. By [5, 11.1.9], $H / k^{\perp}$ is archimedean, so that $|\bar{h}|=0$ modulo $k^{\perp}$, i.e., $h \in k^{\perp}$.

(b) Let $G \leq H$ be coessential so that $H=\bigcup_{\alpha<\omega_{1}} G_{\alpha}(\S 1)$, and let $h \in H$ so $h \in G_{\alpha}$ for some $\alpha$. We proceed by induction on $\alpha$.

If $h \in G_{0}$, then $|h| \leq a$ for some $a \in G$, and then $a^{\perp H} \subseteq h^{\perp H}$.

Let $h \in G_{\alpha}$, and suppose the conclusion of (b) for all elements of $G_{\beta}$, for each $\beta<\alpha$. If $\alpha$ is a limit ordinal, then $G_{\alpha}=\bigcup_{\beta<\alpha} G_{\beta}$, and there is nothing to prove. If $\alpha=\beta+1$, then $h_{n} \rightarrow h$ r.u. for some $\left\{h_{n}\right\}_{n} \subseteq G_{\beta}$, and $\bigcap_{n} h_{n}^{\perp H} \subseteq$ $h^{\perp H}$ by (a). By the induction hypothesis, for each $n$ there is countable $A_{n} \subseteq G$ with $A_{n}^{\perp H} \subseteq h_{n}^{\perp H}$, so that $\left(\bigcup_{n} A_{n}\right)^{\perp H}=\bigcap_{n} A_{n}^{\perp H} \subseteq \bigcap_{n} h_{n}^{\perp H} \subseteq h^{\perp H}$. Invoking induction concludes the proof.

To show that (c) implies (d) in 2.1 , we shall prove the following:

2.3. Proposition. (a) In Arch, for each $G$, there is epicomplete $H$ with $G \leq H$ epic and essential.

(b) For l-groups, if $G \leq H$ is essential, then whenever $A \subseteq G$ has $A^{\perp G}=(0)$, then $A^{\perp H}=(0)$.

(c) If an l-group $H$ has ppp (see $§ 1)$ and $A$ is a countable subset of $H$, then there is countable, pairwise disjoint $B$ with $B^{\perp H}=A^{\perp H}$.

Then we prove (c) implies (d) in 2.1 as follows: Suppose $G$ has a countable subset $A$ with $A^{\perp G}=(0)$. Let $G \leq H$ as in 2.3(a). By $2.3(\mathrm{~b}), A^{\perp H}=(0)$. Since $H$ is epicomplete, it has ppp, so choose $B$ as in $2.3(\mathrm{c})$; then $B^{\perp H}=(0)$. Since $H$ is epicomplete, it is laterally $\sigma$-complete, so we have $u=\bigvee B$. Of course, $(\bigvee B)^{\perp H}=B^{\perp H}$; whence $u$ is a weak unit.

Proof of 2.3. (a) Start with an essential extension $G \leq E$ with $E$ epicomplete. (For example, $E=D(X)$, where $X$ is the extremally disconnected Stone space of the polar algebra of $G . E$ contains $G$ essentially and is divisible, conditionally and laterally complete, hence epicomplete; see [5, 13.4.1] or [7].)

Now factor $G \leq E$ as $G \leq H \leq E$, with $G \leq H$ epic (and automatically essential), and $H$ having no epic extension within $E$. (This is possible on categorical grounds [11,34.4], granted some knowledge of the category Arch [2, p. 3], but also with bare hands: let $H$ be the sub-l-group of $E$ generated by $\bigcup\{K \leq E \mid G \leq K$ is epic $\})$. $H$ is, thus, an "extremal subobject" of epicomplete $E$, and by [3, 4.9] is itself epicomplete.

(b) Easy.

(c) Let $A=\left\{a_{n}: n \in N\right\} \subseteq H$. Without changing $A^{\perp}$, we arrange it that $0 \leq a_{i} \leq a_{i+1}$ for each $i$. Let $a_{0}^{\prime}=a_{0}$. For $i>0$, we have $H=a_{i-1}^{\perp \perp} \oplus a_{i-1}^{\perp}$; let $a_{i}^{\prime}$ be the component of $a_{i}$ in $a_{i-1}^{\perp}$. Then for $i<j, a_{j}^{\prime} \in a_{j-1}^{\perp} \subseteq a_{i}^{\perp} \subseteq a_{i}^{\prime \perp}$, so that $B=\left\{a_{n}^{\prime}: n \in N\right\}$ is pairwise disjoint.

For each $n,\left(\bigvee_{i \leq n} a_{i}^{\prime}\right)^{\perp}=a_{n}^{\perp}: \subseteq$ is clear, and an easy induction shows $\supseteq$. It follows that $B^{\perp}=A^{\perp}$.

\section{THE SIZE OF $H$ OVER $G$}

In Theorem 2.1 , the construction of the $H$ with weak unit occurs in "(c) implies (d)." That construction is a rather natural one (4.4), but one nonetheless 
wonders if some rather simple process might suffice to epically adjoin a weak unit. The results below suggest not.

In case $G \leq H$ has $H$ generated as an $l$-group by $G$ together with finitely (resp. countably) many elements, we say that $H$ is finitely (resp. countably) generated over $G$.

3.1. Theorem. In Arch,

(a) If $G \leq H$ is coessential, $H$ has a weak unit but $G$ does not, then $H$ is not finitely generated over $G$;

(b) If $G \leq H$ is coessential, $H$ has a weak unit but $G$ does not, and also $H$ is laterally $\sigma$-complete and has ppp, then $H$ is not even countably generated over $G$;

(c) If $G$ has any coessential extension with a weak unit, then $G$ has one that is countably generated over $G$.

Remark. Evidently, in (a) and (b) above, "coessential" may be replaced by "epic." In (c), we do not know if "coessential" may be replaced by "epic"; deciding that would seem to require fully coming to grips with the characterization of epics in [2], and that is technically forbidding.

Proof of 3.1. The following shall be used twice.

3.2. Lemma. In abelian l-groups, if $G \leq H$ and $H$ is generated by $G \cup F$, then for each $h \in H$, we have $|h| \leq g+p f$ for some $g \in G, p \in N$, and $f=\bigvee\{|t| \mid t \in T\}$, where $T$ is a finite subset of $F$.

Proof of 3.2. By [5, 2.1.3], $h=\bigwedge_{i} \bigvee_{j}\left(g_{i j}+\sum_{k} n_{i j k} f_{i j k}\right)$ over finite index sets, with the $g$ 's $\in G$, the $n$ 's integers, the $f$ 's $\in F$. It follows that $|h| \leq \bigvee_{i j}\left|g_{i j}\right|+$ $\left(\bigvee_{i j k}\left|n_{i j k}\right|\right) \bigvee_{i j k}\left|f_{i j k}\right| \equiv g+p f$.

Proof of 3.1(a). An embedding $G \leq H$ is called majorizing if whenever $h \in H$, there is $g \in G$ with $|h| \leq g$; clearly, then, if $H$ has a weak unit, so does $G$. Thus, the following implies 3.1(a).

3.3. Proposition. If, in Arch, $G \leq H$ is coessential and $H$ is finitely generated over $G$, then $G \leq H$ is majorizing.

Proof of 3.3. Under the hypothesis, we have $H=\bigcup_{\alpha<\omega_{1}} G_{\alpha}(\S 1)$ and $F$ finite with $G \cup F$ generating $H$. We are to show $H=G_{0}$. Let $k=\bigvee\{|f| \mid f \in F\}$. It suffices to show that $k \in G_{0}$.

If $k \notin G_{0}$, then there is least $\alpha$ such that $k \in G_{\alpha+1} \backslash G_{\alpha}$, and so there is $\left\{h_{n}\right\}_{n} \subseteq G_{\alpha}$ with $h_{n} \rightarrow k$ r.u., say, with regulator $h$. By $3.2, h \leq g+p k$ for some $g \in G, p \in N$, and it follows that $h_{n} \rightarrow k$ regulated by $g+p k$. Thus, there is $n$ with $2 p\left|h_{n}-k\right| \leq g+p k$, and so $2 p k \leq p k$ modulo $G_{\alpha}$. But $0 \leq 2 p k \leq p k \leq 2 p k$ modulo $G_{\alpha}$ implies $k=0$ modulo $G_{\alpha}$. That is, $k \in G_{\alpha}$, a contradiction.

Proof of 3.1(b). Suppose $G \leq H$ is as in the hypothesis. By 2.1, there is countable $\left\{a_{n}: n \in N\right\} \subseteq G$ with $\left\{a_{n}: n \in N\right\}^{\perp H}=(0)$, and since $H$ has ppp, by $2.3(\mathrm{c})$ and its proof, there is countable, pairwise disjoint $\left\{u_{n}: n \in N\right\} \subseteq H$ with $\left\{u_{n}: n \in N\right\}^{\perp H}=(0)$ and $0 \leq u_{n} \leq a_{n}$ for all $n$. Since $H$ is laterally $\sigma$-complete, $u=\bigvee_{n} u_{n}$ is a weak unit in $H$.

Let $F=\left\{f_{n}: n \in N\right\} \subseteq H$. For each $n$, let $h_{n}=u \vee \bigvee_{i \leq n}\left|f_{n}\right|$, so that $u \leq h_{n} \leq h_{n+1}$. We shall find $h>0$ so that $h \nless g+n h_{n}$ for each $g \in G^{+}$and 
$n \in N$. Thus, the conclusion of 3.2 fails for $F$ and $G \cup F$ does not generate $H$.

For each $n, H=u_{n}^{\perp \perp} \oplus u_{n}^{\perp}$, and we let $h_{n}^{n}$ be the component of $h_{n}$ in $u_{n}^{\perp \perp}$. Set $h=\bigvee_{n}(n+1) h_{n}^{n}$. Notice that $h=(n+1) h_{n} \bmod u_{n}^{\perp}$.

Now, whenever $g \in G^{+}$, we have $g^{\perp} \nsubseteq u_{n}^{\perp}$ for infinitely many $n$ (for, if $k$ is biggest with $g^{\perp} \nsubseteq u_{k}^{\perp}$, then $g \vee \bigvee_{n<k} a_{n}$ is a weak unit in $G$ ), and, whenever $g^{\perp} \nsubseteq u_{n}^{\perp}$, we have $u \not \leq g \bmod u_{n}^{\perp}$.

Seeking a contradiction, suppose $h \leq g+p h_{p}$ for some $p$, and $g \in G^{+}$. Choose $n \geq p$ with $g^{\perp} \nsubseteq u_{n}^{\perp}$. We then have $h \leq g+n h_{n}$; thus $h \leq g+$ $n h_{n} \bmod u_{n}^{\perp}$, which means $(n+1) h_{n} \leq g+n h_{n} \bmod u_{n}^{\perp}$, or $h_{n} \leq g \bmod u_{n}^{\perp}$. Hence $u \leq g \bmod u_{n}^{\perp}$, which contradicts the last paragraph.

Proof of 3.1(c). Given $G \leq H$ and $L \subseteq H$, let $G(L)$ stand for the sub-l-group of $H$ generated by $G \cup L$. The following proposition applied to a weak unit $h$ yields $3.1(\mathrm{c})$.

3.4. Proposition. Let $G \leq H$ be coessential and $h \in H$. Then there is countable $L \subseteq H$ such that $G \leq G(L)$ is coessential and $h \in G(L)$.

Proof of 3.4. We shall prove the following weak version of 3.4:

(*) If $G \leq H$ is coessential, and $h \in H$, then there is countable $L \subseteq H$ such that $h \in \operatorname{ak}_{G(L)} G$.

$\left((*)\right.$ is not 3.4 , since $G \leq \mathrm{ak}_{G(L)} G$ need not be coessential, nor countably generated over $G$.)

Granted (*), we prove 3.4: By (*), choose countable $L_{1}$ with $h \in \mathrm{ak}_{G\left(L_{1}\right)} G$. Given countable $L_{n}$, for each $x \in L_{n}$, choose countable $L_{x}$ with $x \in \operatorname{ak}_{G\left(L_{x}\right)} G$ (by (*), again), and put $L_{n+1}=\bigcup\left\{L_{x} \mid x \in L_{n}\right\}$. We then have $x \in \operatorname{ak}_{G\left(L_{n+1}\right)} G$ for each $x \in L_{n}$. By induction, $L_{n}$ is defined for each $n$, and we set $L=$ $\bigcup_{n} L_{n}$. So, $\operatorname{ak}_{G\left(L_{n+1}\right)} G \subseteq \operatorname{ak}_{G(L)} G$ for each $n$, whence $L \subseteq \bigcup_{n} \operatorname{ak}_{G\left(L_{n+1}\right)} G \subseteq$ $\operatorname{ak}_{G(L)} G$, whence $G(L) \subseteq \operatorname{ak}_{G(L)} G$ (since the latter is a sub-l-group containing $G$ and $L)$, and this just says $G \leq G(L)$ is coessential.

We prove (*): Under the hypotheses, $H=\bigcup_{\alpha<\omega_{1}} G_{\alpha}$, so $h \in G_{\alpha}$ for some $\alpha$. We proceed by induction on $\alpha$.

If $h \in G_{0}$, use $L=\{h\}$.

Suppose the conclusion of (*) for all elements of $G_{\beta}$, for every $\beta<\alpha$, and let $h \in G_{\alpha}$. If $\alpha$ is a limit ordinal, already $h \in G_{\beta}$ for some $\beta<\alpha$ and there is nothing to prove. If $\alpha=\beta+1$, then there is $\left\{h_{n}\right\}_{n} \subseteq G_{\beta}$ and $v \in H$ with $h_{n} \rightarrow h$ regulated by $v$. For each $n$, the induction hypothesis provides countable $L_{n}$ with $h_{n} \in \operatorname{ak}_{G\left(L_{n}\right)} G$. Let $L=\bigcup_{n} L_{n} \cup\{v\}$. Then, for each $n$, $\operatorname{ak}_{G\left(L_{n}\right)} G \subseteq \operatorname{ak}_{G(L)} G$, so $\left\{h_{n}\right\}_{n} \subseteq \operatorname{ak}_{G(L)} G$. Since $v \in G(L), h \in \operatorname{ak}_{G(L)} G$ follows. Invoking induction proves $(*)$.

The proof of $3.1(\mathrm{c})$ is concluded.

\section{REMARKS}

4.1. Various aspects of adjoining units to $l$-groups or vector lattices are treated in [6], more recently in [8], and of course some of the representation theorems for archimedean l-groups (such as the one mentioned in the proof of 2.3(a)) have the effect of adjoining a unit. None of that has much to do with this paper.

One may note that $l$-groups with unit stand in some analogy with rings with identity and that adjoining a unit is rather like adjoining an identity to a ring. 
4.2. Regarding rings in another vein, any archimedean $H$ with weak unit $u$ can be Arch-epically embedded in an $f$-ring $H$ with identity $u$ by [10]. Thus, when $G$ "satisfies 2.1 ," one may take an $H$ for 2.1 to be an $f$-ring with identity. As a matter of fact, the $H$ constructed in "(c) implies (d)" is an $f$-ring with identity, by [3, 3.9], and some further argument.

4.3. Our original concept of the present work was as a contribution towards understanding some of the Arch-epicompletions of the $G$ 's satisfying 2.1 , because some of them are epicompletions in "the weak unit category $W$," and $W$-epicompletions are fully understood [4].

That still seems valid, but the central concept of this paper would now appear to be coessentially rather than epicity.

4.4. The $H$ constructed in "(c) implies (d)" of 2.1 is an essential epicompletion of $G$. It is shown in [12] that such a thing is unique over $G$ and lifts complete homomorphisms to epicomplete targets. (The analogous facts in $W$ are described in [4].)

4.5. One wonders whether, when $G$ satisfies 2.1 , every epicompletion of $G$ has weak unit. The answer is "no," but the example would carry us too far afield. However, for each $G$, there is an epicompletion $\beta G$ that lifts all homomorphisms to epicomplete targets [3], and $\beta G$ has weak unit if and only if every epicompletion of $G$ has weak unit [12]. This example depends on an analysis of $\beta G$ [12].

\section{REFERENCES}

1. M. Anderson and T. Feil, Lattice-ordered groups, an introduction, Reidel, Dordrecht, Boston, Lancaster, and Tokyo, 1988.

2. R. N. Ball and A. W. Hager, Characterization of epimorphisms in archimedean latticeordered groups and vector lattices, Lattice-Ordered Groups, Advances and Techniques (A. Glass and C. Holland, eds.), Chapter 8, Kluwer Acad., Dordrecht, Boston, and London, 1989.

3. __ Epicomplete archimedean l-groups and vector lattices, Trans. Amer. Math. Soc. 322 (1990), 459-478.

4. _ Epicompletion of archimedean l-groups and vector lattices with weak unit, J. Austral. Math. Soc. Ser. A 48 (1990), 25-56.

5. A. Bigard, K. Keimel, and S. Wolfenstein, Groupes et anneaux réticulés, Lecture Notes in Math., vol. 608, Springer, Berlin, Heidelberg, and New York, 1977.

6. B. Brainerd, On the embedding of a vector lattice in a vector lattice with weak unit, Nederl. Akad. Wetensch. Proc. Ser. A 63 (1960), 25-31.

7. P. F. Conrad, The essential closure of an archimedean lattice-ordered group, Proc. London Math. Soc. 38 (1971), 151-160.

8. P. F. Conrad and J. Martinez, Complemented lattice-ordered groups, Indag. Math. (N.S.) (3) 1 (1990), 281-298.

9. A. M. W. Glass and W. C. Holland, Lattice-ordered groups, advances and techniques, Kluwer Acad., Dordrecht, Boston, and London, 1989.

10. A. W. Hager and L. C. Robertson, On the embedding into a ring of an archimedean l-group, Canad. J. Math. 31 (1979), 1-8.

11. H. Herrlich and G. Strecker, Category theory, Allyn and Bacon, Boston, MA, 1973; reprinted by Heldermann-Verlag, Berlin, 1979. 
12. A. Kizanis, Epicompletions of archimedean lattice-ordered groups, Doctoral Thesis, Wesleyan University, 1991.

13. W. Luxemburg and A. Zaanen, Riesz spaces, Vol. 1, North-Holland, Amsterdam and London, 1971.

(R. N. Ball) Department of Mathematics and Computer Science, University of Denver, University Park, Denver, Colorado 80208

E-mail address: rball@Math.cs.du.edu

(A. W. Hager and A. Kizanis) Department of Mathematics, Wesleyan University, Middletown, Connecticut 06459

E-mail address: ahager@eagle.wesleyan.edu

Current address, A. Kizanis: Department of Mathematics, Western New England College, Springfield, Massachusetts 01119

E-mail address: akizanis@wnec.bitnet 